\title{
INTER-SATELLITE COMMUNICATIONS CONSIDERATIONS AND REQUIREMENTS FOR DISTRIBUTED SPACECRAFT AND FORMATION FLYING SYSTEMS
}

\author{
Carl F. Kwadrat*, William D. Horne*, Bernard L. Edwards** \\ *ITT Industries, Advanced Engineering and Sciences, 1761 Business Center Drive, Reston VA 20190 \\ **Associate Head, Microwave Systems Branch, NASA Goddard Space Flight Center,
} Greenbelt, MD 20771

\begin{abstract}
In order to avoid selecting inadequate inter-spacecraft cross-link communications standards for Distributed Spacecraft System (DSS) missions, it is first necessary to identify cross-link communications strategies and requirements common to a cross-section of proposed missions. This paper addresses the cross-link communication strategies and requirements derived from a survey of 39 DSS mission descriptions that are projected for potential launch within the next 20 years. The inter-spacecraft communications strategies presented are derived from the topological and communications constraints from the DSS missions surveyed. Basic functional requirements are derived from an analysis of the fundamental activities that must be undertaken to establish and maintain a cross-link between two DSS spacecraft. Cross-link bandwidth requirements are derived from high-level assessments of mission science objectives and operations concepts. Finally, a preliminary assessment of possible cross-link standards is presented within the context of the basic operational and interoperability requirements.
\end{abstract}

\section{Introduction}

DSS missions consist of multiple space platforms that act in consort gathering data to achieve a single science objective. The spacecraft gather science data from different spatial and temporal perspectives for reduction into a single format that is more informative than that provided by any of the individual platforms within the distribution. Examples of this are spacecraft with radiation sensors that form the synthetic apertures that are the basis of optical and radio-based interferometers, and the multi-perspective images that provide time-sequenced images of certain natural phenomena. Two distinct categories of DSS missions exist based on the location where the data is reduced [1]. Ground based data reduction requires that all of the science data be transferred to the ground via an independent space-ground link capability that is associated with each member of the distribution. Space based data reduction requires that one or more spacecraft collect and process the data from the other members of the distribution. This alternative requires that communications cross-links exist between the spacecraft that supports the transfer of the science data amongst the members. In such circumstances, the data maybe entirely processed, partially processed, or just repackaged prior to being transferred to the mission ground segment. The choice of data transfer alternatives depends on the mission design. This paper will concentrate on DSS missions that require cross-links to meet their science operations objectives.

\section{Cross-Link Communications Strategies}

Cross-link based Distributed Spacecraft Missions can be divided into two main categories: constellation and formation flying missions. Constellations differ from formation flying missions in the manner in which the members of these distributions interact with each other. Constellations are formed from a group of spacecraft orbiting a planetary body or the sun. These constellation topologies are characterized by groupings of spacecraft in circular or elliptical orbits about a central body. Their positions are 
routinely maintained by station keeping operations that are governed by navigation information obtained from the ground segment or on-board navigation sensors. Their relative positions are maintained by their nearest neighbors for cross-link antenna pointing purposes. On the other hand, spacecraft in formation flying missions exchange their positional information across the cross-links so that feedback based corrections to their locations can be made in order to keep the structure of the formation within the geometrical tolerances specified by the mission objectives. The degree navigational coupling between members of the formation is determined by the level of precision needed to maintain the spacecraft within the geometrical tolerances specified by the mission objectives.

The elementary building blocks of constellations are spacecraft orbiting a central body, while that of the formation flying mission are spacecraft with nearly fixed positions with respect to each other. Figure 1 shows two extremes in the communications architectures or topologies associated with formation flying missions.

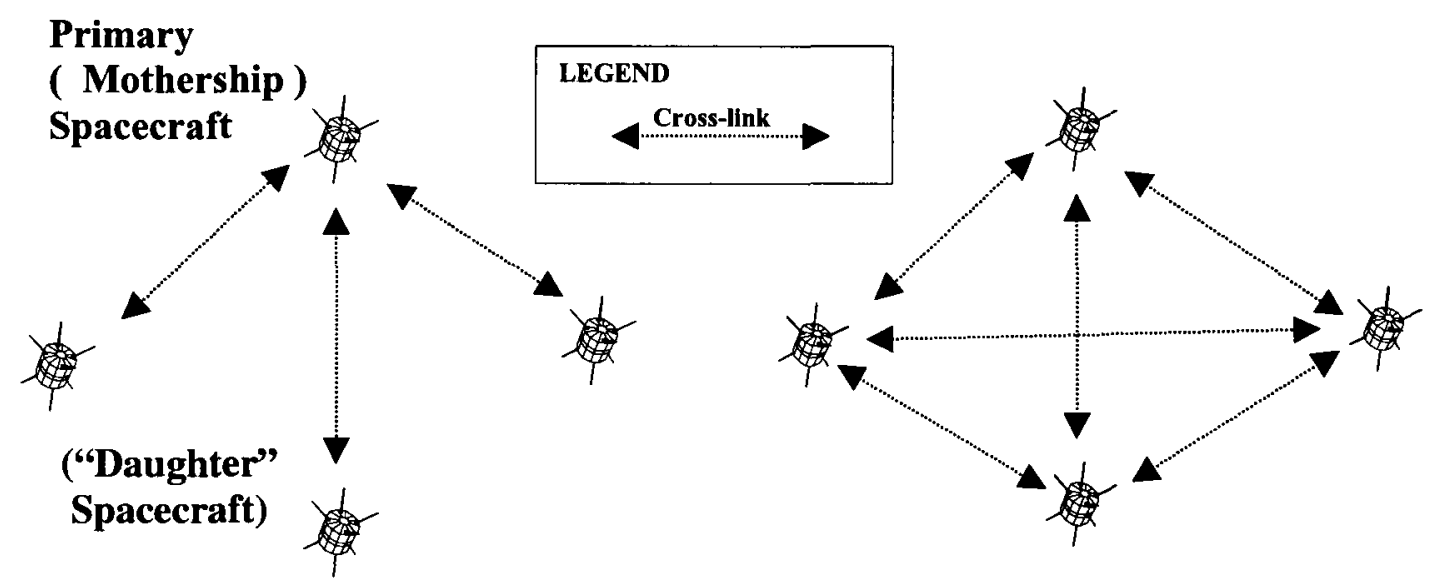

\section{Centralized (Star) Topology \\ Distributed (Ad hoc)Topology \\ Figure 1: Basic Formation Flying Cross-Link Communications Topologies}

The Centralized (Star) Topology is formed from a "mothership" and any number of "daughter" spacecraft. The "mothership" maintains a master-to-slave relationship with respect to each of its "daughters". The Distributed (Ad hoc) Topology does not have the master-slave characteristics of the Centralized Topology. Instead, every member of the distribution has equality in operations with respect to every other member. Each spacecraft is responsible for its own navigation control determination based on the assessment of the collective positional data that it receives from all of the other spacecraft via the cross-links. Science data from each may be forwarded to a single spacecraft via the cross-links or downloaded individually to the ground segment.

The inter-spacecraft separation and the number of spacecraft in the distribution plays an important role in determining the cross-link communications strategies that can be adopted by a mission. For instance, if hundreds of low powered nanosats are used in a mission and the mission is spread out over a large enough region of space, each nanosat may not have enough power to form a cross-link with every other nanosat. As such, a pure Distributed Topology cannot be realized and hybrid topologies may be an automatic constraint for the mission. 
DSS missions with relatively fixed geometries present relatively simple solutions for cross-link antenna beam patterns, especially when the number of spacecraft is small. These geometries lend themselves to fixed antennas. At the opposite extreme, a constellation in several highly elliptical orbits about a planetary body (e.g., magnetosphere mapping missions) introduces significantly more complexity to the cross-link solution for the mission. This is due to the fact that the overall geometry of the distribution is in a constant state of change along with the relative position of the orbiting spacecraft. Low-gain fixed antenna beams severely limit the amount of cross-link contacts that can be made between the spacecraft. Steerable beam high-gain antennas may be required to achieve a significant increase in the number of cross-link services that can be realized over a given period since each spacecraft can become a rapidly moving target with respect to any other spacecraft. This requirement adds significantly more complexity to the design of the cross-link communications system than that of a nearly "rigid" spacecraft distribution. Differences in orbital periods drives the extreme changes in the relative ranges of the spacecraft that can change the cross-link power requirements of the cross-link signal from near to far distances of separation. Dynamic cross-link network routing strategies with multiple hops may be required if low powered nanosats are required to communicate over distances that are greater than their cross-link transmit power can support. In these instances, the on-board dynamic routing capabilities will be significant in comparison to relatively "rigid" distributions.

The presence of multiple cross-links in a Distributed Spacecraft Mission requires that a protocol be enforced such that members of the distribution do not interfere with each other while attempting to communicate via the cross-links. There are five basic Multiple Access (MA) methods that available to avoid interference between cross-links. These consist of time, frequency, code, spatial separation, and collision detection based resource allocations, or a combination of techniques. Table 2 describes these basic multiple access techniques along with their advantages and disadvantages.

Hybrid versions of the five MA methods shown in Table 1 are possible. All of these MA methods coupled with the cross-link architectural constraints described above to provide compound restrictions on the cross section of cross-link system types considered.

Table 3 summarizes the cross-link communications system requirements based on the extremes in the topological characteristics of the DSS. The first column of Table 3 describes the relative distance of separation of the spacecraft within the topology. Planned missions over the next twenty years have spacecraft separations ranging from distances on the order of kilometers to millions of kilometers. The second column describes the relative degree to which a distribution can maintain its geometrical form under the influence of orbital gravitational dynamics. Mission topologies near planetary bodies undergo large deformations due to the effects of non-uniform gravitational forces and orbital geometric constraints on their spacecrafts' relative orbital motions. For the most part, Deep Space missions avoid this type of geometrical distortion when their spacecraft are at planetary distances from the sun. The last column lists the cross-link requirements that are attributed to missions characterized by the constraints imposed by the first two columns. Each of the MA techniques listed in parentheses in the last column suggest that any one or some combination of controlled access for that topology and antenna/transmitter would be reasonable choices for cross-link implementation without any other constraints being specified at this time. 
Table 2 Basic Cross-Link Multiple Access Methods

\begin{tabular}{|c|c|c|c|}
\hline $\begin{array}{c}\text { Classic } \\
\text { Multiple } \\
\text { Access } \\
\text { Method }\end{array}$ & Basic Characteristics & Advantages & Disadvantages \\
\hline $\begin{array}{l}\text { Time } \\
\text { Division } \\
\text { Multiple } \\
\text { Access } \\
\text { (TDMA) }\end{array}$ & $\begin{array}{l}\text { Unique operations time slots } \\
\text { must be assigned to each } \\
\text { cross-link } S / C \\
\text { Each cross-link transaction in } \\
\text { the distribution limited to the } \\
\text { assigned time slot }\end{array}$ & $\begin{array}{l}\text { Single frequency needed for } \\
\text { implementation for all cross- } \\
\text { links } \\
\text { Low cost cross-link solution } \\
\text { due to replicate cross-link } \\
\text { design }\end{array}$ & $\begin{array}{l}\text { Cross-Link transmissions must occur } \\
\text { one cross-link at a time } \\
\text { Time synchronization needed between } \\
\text { all distribution S/C } \\
\text { Propagation delay corrections must be } \\
\text { applied when cross-link signal path } \\
\text { lengths vary in order to avoid signal } \\
\text { collisions } \\
\text { The greater the number of S/C, the } \\
\text { longer the duty interval for cross-link } \\
\text { transmissions by a given S/C resulting } \\
\text { in lower the overall data throughput for } \\
\text { the distribution } \\
\text { Changing S/C distances of separation } \\
\text { requires dynamic assessments of time } \\
\text { slot allocations to compensate for } \\
\text { variable signal delays }\end{array}$ \\
\hline $\begin{array}{l}\text { Frequency } \\
\text { Division } \\
\text { Multiple } \\
\text { Access } \\
\text { (FDMA) }\end{array}$ & $\begin{array}{l}\text { Unique frequencies needed } \\
\text { for each cross-link }\end{array}$ & $\begin{array}{l}\text { Multiple cross-link } \\
\text { transmissions can occur } \\
\text { simultaneously }\end{array}$ & $\begin{array}{l}\text { One center frequencies needed for each } \\
\text { cross-link implementation } \\
\text { The larger the distribution of S/C the } \\
\text { greater the frequency band allocation } \\
\text { required for the mission } \\
\text { Increased cost due to frequency } \\
\text { variation in the cross-link design }\end{array}$ \\
\hline $\begin{array}{l}\text { Code } \\
\text { Division } \\
\text { Multiple } \\
\text { Access } \\
\text { (CDMA) }\end{array}$ & $\begin{array}{l}\text { Cross-Link signal must be } \\
\text { randomly spread across a } \\
\text { portion of the frequency band } \\
\text { via PN code }\end{array}$ & $\begin{array}{l}\text { Multiple cross-link } \\
\text { transmissions can occur } \\
\text { simultaneously } \\
\text { Relative range measurements } \\
\text { for the cross-link can be made } \\
\text { simultaneously with } \\
\text { communication operations }\end{array}$ & $\begin{array}{l}\text { Total number of simultaneous cross-link } \\
\text { transmissions is limited by the CDMA } \\
\text { code noise floor } \\
\text { Complex signal processing needed for } \\
\text { implementation } \\
\text { Complexity in design adds to } \\
\text { communications system cost }\end{array}$ \\
\hline $\begin{array}{c}\text { Random } \\
\text { Access } \\
\text { (RA) } \\
\text { (e.g., } \\
\text { ALOHA) }\end{array}$ & $\begin{array}{l}\text { Cross-Link transmission must } \\
\text { be attempted only when no } \\
\text { other cross-link operations are } \\
\text { detected } \\
\text { Attempt at a cross-link } \\
\text { transmission during a } \\
\text { detected transmission of } \\
\text { another cross-link must be } \\
\text { postponed by a random } \\
\text { amount of time until a } \\
\text { reattempt can be made }\end{array}$ & $\begin{array}{l}\text { Single frequency needed for } \\
\text { implementation for all cross- } \\
\text { links } \\
\text { Low cost cross-link solution } \\
\text { due to replicate cross-link } \\
\text { design }\end{array}$ & $\begin{array}{l}\text { Transmissions must occur one cross- } \\
\text { link at a time limiting data throughput } \\
\text { The greater the distance between S/C, } \\
\text { the greater the likelihood of cross-link } \\
\text { collisions due to propagation delays } \\
\text { masking ongoing cross-link session } \\
\text { startups } \\
\text { The greater the number of S/C, the more } \\
\text { likely that postponements of cross-link } \\
\text { transmissions thus reducing overall data } \\
\text { throughput within the distribution }\end{array}$ \\
\hline $\begin{array}{l}\text { Spatial } \\
\text { Access } \\
\text { (SA) }\end{array}$ & $\begin{array}{l}\text { High gain antennas isolate } \\
\text { beams and prevent cross-link } \\
\text { interference between different } \\
\text { cross-links }\end{array}$ & $\begin{array}{l}\text { Continuous cross-link } \\
\text { services can take place } \\
\text { simultaneously without } \\
\text { interference constraints }\end{array}$ & $\begin{array}{l}\text { Cost of cross-link system increases } \\
\text { significantly due to cost of high gain } \\
\text { antenna system } \\
\text { Cost of cross-link system adds } \\
\text { significant cost to antenna controller if } \\
\text { steerable beams are required }\end{array}$ \\
\hline
\end{tabular}


Table 3 DSS Cross-Link Requirements Imposed by the Extremes in Physical Topological Characteristics of Different DSSs

\begin{tabular}{|c|c|c|}
\hline $\begin{array}{c}\text { Inter-Spacecraft } \\
\text { Distances }\end{array}$ & $\begin{array}{c}\text { Flexibility of } \\
\text { Topological Form }\end{array}$ & DSS Cross-Link Requirements \\
\hline \multirow[t]{2}{*}{ Small } & Low & $\begin{array}{ll}\text { - } & \text { Fixed beam antenna } \\
\text { - } & \text { Low gain antenna with controlled access (TDMA, FDMA, CDMA, andor RA) } \\
\text { - } & \text { Low power transmitter }\end{array}$ \\
\hline & High & $\begin{array}{l}\text { - } \text { Switching among fixed beam antennas or a steerable beam antenna } \\
\text { - } \\
\text { - } \quad \text { Low gain antenna with controlled access (TDMA, FDMA, CDMA, and/or RA) } \\
\end{array}$ \\
\hline \multirow[t]{2}{*}{ Large } & Low & $\begin{array}{l}\text { Fixed beam antenna } \\
\text { High gain antenna, low power transmitter, and controlled access (TDMA, } \\
\text { FDMA, CDMA, RA, and/or SA) } \\
\text { Low gain antenna with controlled access (TDMA, FDMA, CDMA, and/or RA) } \\
\text { and high power transmitter }\end{array}$ \\
\hline & High & $\begin{array}{l}\text { - Steerable beam high gain antenna and low power transmitter, and controlled } \\
\text { access (TDMA, FDMA, CDMA, RA, and SA), or } \\
\text { Fixed beam, low gain antenna, high power transmitter, and controlled access } \\
\text { (TDMA, FDMA, CDMA, and/or RA) }\end{array}$ \\
\hline
\end{tabular}

\section{Cross-Link Communications System Requirements}

Functional analysis provides a structured approach to establishing a framework within which related requirements can be gathered. Figure 2 shows a context diagram of a cross-link communications system that is represented by a circle. The boundary of the system encompasses all of the functionality needed to establish and maintain a cross-link connection between two spacecraft. Information flows into and out of the system are represented as arrows. The external entities that interface to the system are the Cross-Link Partner S/C, the On-board Navigation Processor, the On-board Communications Event Scheduler, the Onboard Communications Configuration Manager, and the OSI Transport Layer.

Figure 3 shows a high-level requirements model in terms of a functional decomposition of a cross-link communications system shown in Figure 2. Each of the major functions is represented by a circle and the arrows between circles represents the flow of information among the functions in the system. The external information flows shown in Figure 2 are represented by arrows connecting the functions to the external entities shown in Figure 3. Each function transforms one or more input information flows into output flows that are supplied to other functions or the entities external to the cross-link communications system. The circles represent the top-level cross-link requirements areas that lend themselves to the identification of individual functional and performance requirements that flow from the expected behavioral characteristics of each functional area. Carrying out the process of identifying system-level requirements leads to at least 100 system-level functional and performance requirements for the generic cross-link DSS. 


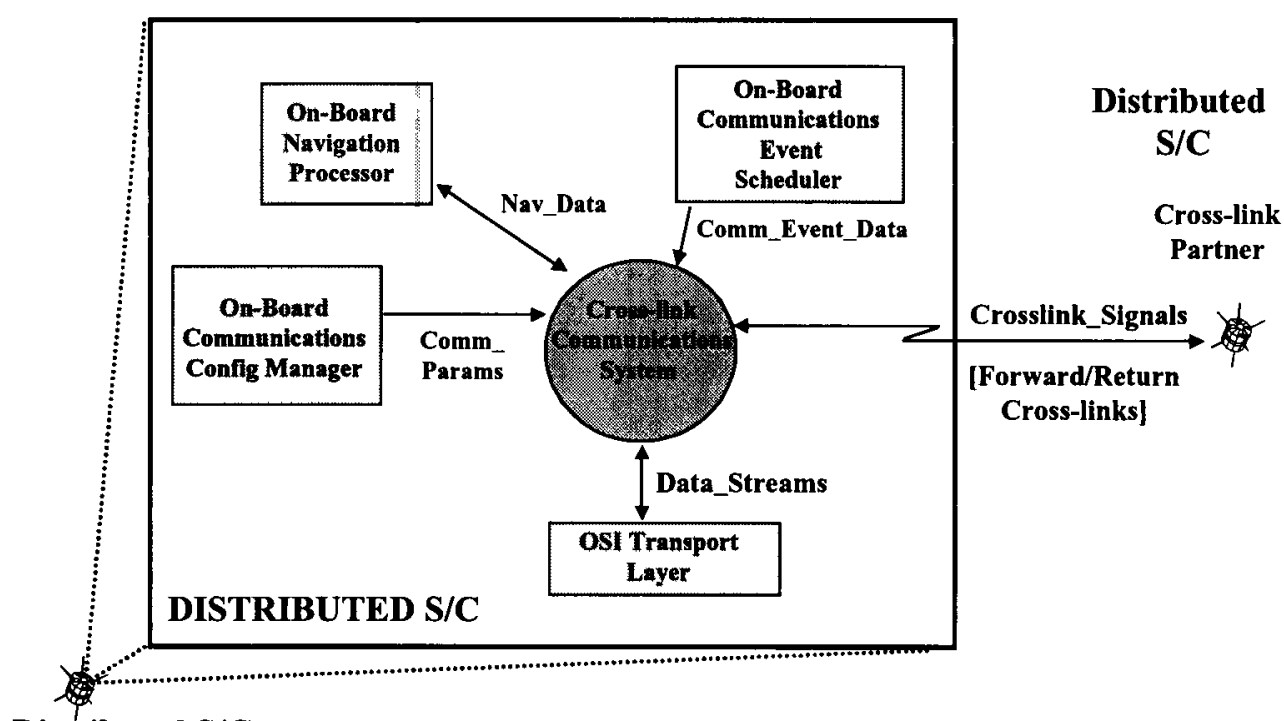

Distributed S/C

Figure 2 Cross-link Communications System Context Diagram

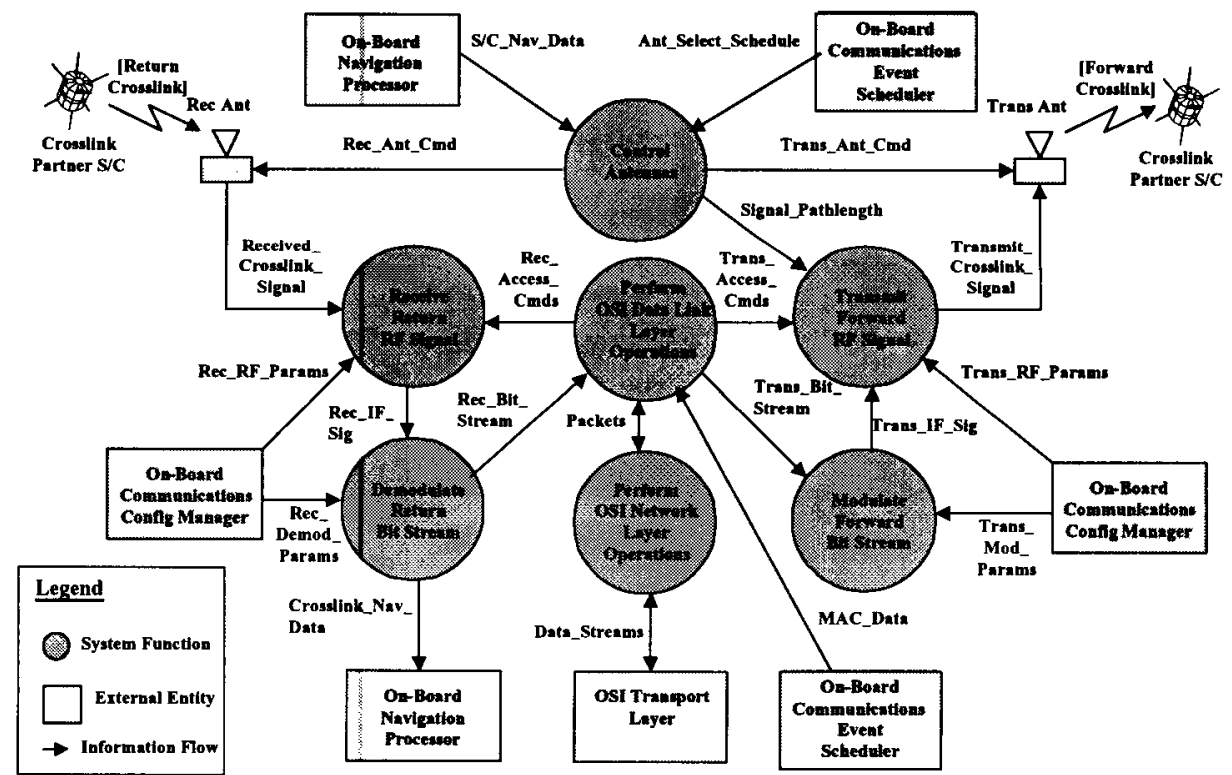

Figure 3 Cross-Link Communications System Functional Requirements Model 
The functions shown in Figure 3 can be grouped into antenna control, physical layer, data link layer and network layer functions. Antenna control fulfills the requirements associated with selection, control, and scheduling of the on-board cross-link antennas. Those functions associated with the modulation of data, the transmission of RF signals, the receipt of RF signals, and the demodulation of data represent those requirement areas traditionally associated with transceivers and the physical layer of a network communications system. The data link function requirements manage the flow and ensure reliable data block exchanges over the cross-links. The network function requirements cover the routing of datagrams to and from the payload equipment and the cross-link.

DSSs lend themselves to internal cross-link networking operations as well as external networking interface operations to other missions and the ground based Internet extended into space. DSSs with very rigid distribution forms and a large number of spacecraft lend themselves to situations where the distributions could be segmented into both Centralized and Distributed Topologies forming a hybrid topology. For example, a small subset of spacecraft in the formation may be designated as "mothership" with the remaining spacecraft becoming their "daughters" thus forming a series of independent Centralized Topologies within the overall mission distribution. The "motherships" in turn could operate as a Distributed Topology amongst themselves. Similarly, the "motherships" could in turn become the "daughters" of one "great grandmother" to form a hierarchical multileveled structure of nested Centralized Distributions. Hybrid topologies can lend themselves to mixtures of cross-link networking architectures that consist of Local Area Networks (LANs) for the Centralized Topologies and Wide Area Networks (WANs) for the higher level Distributed or Centralized Topologies.

Cross-link communications systems will be required to have a Network Layer that provides the routing capabilities within the LAN and WAN perspective. Nearly rigid distributions will be able to use static routing tables to distribute datagrams within the DSS. At the other extreme, highly flexible DSSs will be required to have dynamic routing tables that take into account the constantly changing geometry of the distribution in order determine the best path through the distribution.

The scheduling of services becomes more demanding on the cross-link communications system resources as the DSS increases in the number of spacecraft and the need for simultaneous cross-link operations grows. Large Distributed Formation Flying Topologies with cross-link spacecraft pairs formed from most or all of the members of the distribution are especially sensitive to this growth in operations complexity due to the number of crosslink that must be formed by each spacecraft. Large hierarchical Centralized Formation Flying Topologies are the least impacted. The design of the "mothership" crosslink communication system will be more complicated than that of the "daughters", but the partitioning of the large distribution into smaller formations of Centralized Topologies make design of "mothership" significantly less complicated than that of any of the members of the highly connected Distributed Formation Flying Topologies. The tradeoff is that the rate of information flow within the Centralized Topology will be less than that which is achievable within Distributed topology since latency producing multiple hops will be required to move datagrams across the clustered LANs of the Centralized Topology while the transfers are direct between end points within the Distributed Topology.

\section{Cross-link Bandwidth and Spectrum Requirements}

Crosslinks are expected to carry the following four types of data between distributed spacecraft: science, navigation, command, and spacecraft health data. The volume and kinds of data depend on the objectives of the mission. For example, formation flying missions requiring tight coupling between the spacecraft will experience frequent transfers of navigation and command data on the cross-links within the distribution. Centralized formation flying missions collecting science imaging data may need to frequently transfer high volumes of science data from collector spacecraft across the crosslinks for 
preprocessing on a "mothership" prior to relaying the reduced data to the ground segment on a spaceground link. Spacecraft on-board storage will help determine the frequency and volume of science data transfers thereby specifying the crosslink channel capacity requirements for the mission. Since constellation operations do not require precision autonomous navigation operations, navigation will be a low frequency data transfer operation for this type of DSS. The anticipated maximum cross-link data rates for navigation, command, and health data are on the order of $1 \mathrm{kbps}$ each. Science data rates may be up to $10 \mathrm{Mbps}$ with the possibility of higher data rates if data bursting is required.

A survey of distributed spacecraft missions for the period from 2001 to 2020 identified 39 missions currently in the planning stages and analyzed the spectrum resources necessary to conduct operations [2]. The basic operational concepts of the missions were taken into account to guide the assessment process. Using the identified set of missions, the study projected the expected number of crosslinks assuming mission characteristics such as a maximum lifetime of 10 years. Simultaneous cross-link transmissions were counted with an appropriate factor included to reduce the worst-case possibility of every cross-link operating simultaneously by an appropriate percentage of the maximum number of possibilities. Table 4 shows the estimated maximum number of possible simultaneous near-Earth cross-link transmissions as a function of the estimated mission launch date grouped into the three major mission types discussed above.

Based on the survey of distributed spacecraft missions and an assessment of data needs, the amount of radio frequency bandwidth required for all space and earth science crosslinks at any given time over the next 20 years is less than $120 \mathrm{MHz}$ with a time varying need as indicated in Table 5. Estimating the total bandwidth required for space and earth science distributed spacecraft missions using crosslinks requires a simple calculation using the probable number of simultaneously operational crosslinks, the probabilities that mission crosslinks need narrow, medium, or wide bandwidth communication capabilities, and the associated maximum data rate with the narrow, medium, or wide bandwidth capability. Equation 1 defines the method of estimating the total maximum data rate from which the total bandwidth requirements for all of the distributed spacecraft missions was derived. The subscript $j$ corresponds to the crosslink architecture type $(j=1,2$, and 3 corresponds to constellation, centralized formation, and distributed formation, respectively). The subscript $i$ corresponds to the bandwidth category ( $i=1,2$, and 3 corresponds to narrow, medium, and wide bandwidths, respectively). The probability that a particular maximum bandwidth will be required for a given architecture is represented by $\mathrm{p}_{\mathrm{ij}}$. $\mathrm{N}_{\mathrm{i}}$ is a function of the number of missions within each distributed spacecraft architecture, the number of possible crosslinks that can be formed, and the likelihood that crosslink multiple access techniques for a given mission will support simultaneous transmissions. Additional details can be found in [Ref].

$$
\text { Total Max Data Rate }=\sum_{j=1}^{3} N_{j} \times\left[\sum_{i=1}^{3} p_{i j} \times M D R i\right]
$$

There are many frequency bands (from $400 \mathrm{MHz}$ to over $100 \mathrm{GHz}$ ) allocated to services defined by the regulatory community in which a crosslink system that transfers information between distributed spacecraft shares characteristics including: Earth Exploration-Satellite; Space Operation; Space Research; Inter-Satellite; and, Radionavigation and Radionavigation-satellite service (for signals transmitted solely for navigational purposes). Based on the bandwidth assessment and regulatory review, the space and earth science community does not need to pursue new frequency allocations for non-relay inter-satellite communications at this time since existing allocations should provide sufficient spectrum to meet expected demands through 2020 . To satisfy regulatory considerations and to promote interoperability, distributed spacecraft missions implementing inter-satellite communications and navigation exchange (crosslinks) should seek assignments in the frequency bands listed in Table 6. Each of these bands will have different characteristics affecting the operations of distributed spacecraft missions. 
Table 4: Estimated Maximum Number of Possible Simultaneous Cross-Link Transmissions in Earth Orbit as a Function of Mission Launch Date

\begin{tabular}{|l|c|c|c|}
\hline Operational Intervals & Constellations & $\begin{array}{c}\text { Centralized } \\
\text { Formations }\end{array}$ & $\begin{array}{c}\text { Distributed } \\
\text { Formations }\end{array}$ \\
\hline 2001 to 2005 & 6 & 2 & 13 \\
\hline 2006 to 2010 & 7 & 4 & 39 \\
\hline 2011 to 2015 & 7 & 7 & 13 \\
\hline 2016 and beyond & 7 & 12 & 25 \\
\hline
\end{tabular}

Table 5: Estimated Space \& Earth Science Crosslink Radio Frequency Bandwidth Needed

\begin{tabular}{|l|c|}
\hline \multicolumn{1}{|c|}{ Operational Intervals } & $\begin{array}{c}\text { Est. Max Bandwidth } \\
\text { Requirements For } \\
\text { Operational Missions } \\
\text { (MHz) }\end{array}$ \\
\hline 2001 to 2005 & 27.3 \\
\hline 2006 to 2010 & 90.7 \\
\hline 2011 to 2015 & 107.3 \\
\hline 2016 and beyond & 116.6 \\
\hline
\end{tabular}

Table 6: Preliminary Preferred Frequency Bands with Allocations Allowing Space-to-Space Communications

\begin{tabular}{|c|l|l|}
\hline Band & \multicolumn{1}{|c|}{ Frequency Band } & \multicolumn{1}{c|}{ Allocation Status* } \\
\hline \multirow{3}{*}{$\mathrm{S}$} & $2025-2110 \mathrm{MHz}$ & $\begin{array}{l}\text { SPACE OPERATION } \\
\text { EARTH EXPLORATION SATELLITE } \\
\text { SPACE RESEARCH }\end{array}$ \\
\cline { 2 - 3 } & $2200-2290 \mathrm{MHz}$ & $\begin{array}{l}\text { SPACE OPERATION } \\
\text { EARTH EXPLORATION SATELLITE } \\
\text { SPACE RESEARCH }\end{array}$ \\
\hline \multirow{2}{*}{$\mathrm{Ku}$} & $14.5-15.35 \mathrm{GHz}$ & $\begin{array}{l}\text { Space Research (The 14.5-15.35 GHz band is on the agenda of } \\
\text { WRC-03 for possible upgrade to primary status) }\end{array}$ \\
\hline \multirow{2}{*}{$\mathrm{Ka}$} & $22.55-23.55 \mathrm{GHZ}$ & INTER-SATELLITE \\
\cline { 2 - 3 } & $25.25-27.5 \mathrm{GHz}$ & INTER-SATELLITE \\
\hline
\end{tabular}

*Primary allocations listed by CAPITAL letters; secondary in lower case.

\section{Standards for Cross-Link Implementations}

The adoption of cross-link standards is important when mission interoperability such as that envisioned from a generalized Sensor Web perspective are taken into account. The sharing of information between missions distributed space missions or any other type of mission demands uniformity in waveforms and protocols among the participating members. Common waveforms and network protocols can allow science data and scientific event alerts to be shared among missions. Developing cross-link standards from scratch can be a long process and costly to implement. 
Standards for cross-link implementations are in principle implicit in the CCSDS Proximity-1 Space Link Protocol draft recommendations. This standard has not been implemented so that development of products suitable for low cost cross-link applications is not currently available. However, wireless industry standards promise to meet at lot of the requirements for cross-link applications and they are rapidly being implemented in terrestrial applications. A number of wireless LAN standards already exist and a high level assessment of them was made within the context of 39 distributed spacecraft missions surveyed. The wireless standards reviewed are: IEEE 802.11, 802.11a, 802.11b, 802.15, 802.16, Bluetooth, and HomeRF. The value of these standards lies in the availability of working system components and the cost savings that would be associated with the acquisition of cross-link components. They offer the same automatic, on-demand flexibility needed by large distributions to accommodate new members as the topology evolves towards its maximum size. Since COTS components are not radiation hardened, development efforts for cross-link applications would eventually require component upgrades for space environments. The wireless standards are being developed to accommodate short range, low power, and omni directional antenna coverage to deal with home, office, or metropolitan area spatial constraints. The antenna and power specifications for these standards would have to be extended to meet the topological related requirements of most of the distributed spacecraft missions surveyed. More work is required to go beyond this preliminary assessment of applicable cross-link standards.

\section{Conclusions}

Adopting a cross-link standard should be thought out carefully from a perspective that encompasses the scope of future DSS missions. Based on a preliminary survey of inter-spacecraft communications strategies and the analysis of system-level functions, it is possible to establish a foundation of cross-link communications requirements that span the range of DSS mission applications. By-products of this process are cross-link bandwidth estimates, spectrum allocation assessments, and insights into interspacecraft communications operations assessments. This process can provide a top-down, disciplined approach of arriving at an acceptable cross-link communications standard that satisfies a broad range of capabilities included in a wide range of DSS missions. Future work should consist of a refinement of this process with the intention of providing a strong basis for accepting existing or developing new cross-link communications standards and for defining distributed spacecraft mission operations approaches.

\section{References}

[1] Kul Bhasin and Jeffrey L. Hayden, "Space Internet Architectures and Technologies for NASA Enterprises," 2001 IEEE Aerospace Conference, Big Sky, MT, 10-17 March 2001

[2] Goddard Space Flight Center, Distributed Spacecraft Crosslink Study Part 1: Spectrum Requirements and Allocation Survey Report and Recommendations, v1.0, 5 June 2002 